\title{
Optimization of compression formulation and load of food-grade tracers for grain traceability using central composite design
}

\author{
Liang Kun, Zhang Lingling, Lu Wei, Cedric Sean Okinda, Shen Mingxia* \\ (College of Engineering/Key Laboratory of Intelligent Equipment for Agriculture of Jiangsu Province,
} Nanjing Agricultural University, Nanjing 210031, China)

\begin{abstract}
Food-grade tracers have been developed as an identification technology for grain traceability from original harvest to final destination for transportation. The characteristics of food-grade tracers must be able to satisfy the environmental demands for grain traceability. To optimize the food-grade tracer production process, the effects of direct compression formulation and load on the mechanical characteristics were studied using response surface methodology (RSM) with central composite design (CCD). Among the four tested formulations, Formulations \#2 (consisting of 35.00\% lactose 100 mesh, $64.50 \%$ microcrystalline cellulose 102 and $0.50 \%$ magnesium stearate) and \#4 (consisting of 38.00\% lactose 100 mesh, $50.00 \%$ microcrystalline cellulose $102,11.00 \%$ pregelatinized starch and $1.00 \%$ magnesium stearate) were selected for tracer production based on their physical properties as powders. The value of Carr's flowability index was 68 for both Formulations $\# 2$ and \#4, which was the highest among all the formulations. Therefore, Formulations \#2 and \#4 also had the best powder flowability. The magnesium stearate ratio (1.00\%-3.00\%) and pressure (6.00-16.00 kgf) were used as independent variables to detect changes in the breaking rate, peak shear force and friction coefficient of tracers compressed by the selected formulations. The optimal production parameters could be achieved at a magnesium stearate ratio of $2.25 \%$ and pressure of $16.00 \mathrm{kgf}$ for Formulation \#2 and at a magnesium stearate ratio of $1.02 \%$ and pressure of $16.00 \mathrm{kgf}$ for Formulation \#4. Under these optimal conditions, the tracers had good impact characteristics (breaking rate), compression characteristics (peak shear force) and frictional characteristics (friction coefficient). Moreover, Formulation \#2 was more suitable for production because compared to Formulation \#4, its breaking rate and friction coefficient values were lower, and its peak shear force value was higher.
\end{abstract}

Keywords: grain traceability, food-grade tracer, optimization, food safety, formulation, mechanical property, central composite design DOI: $10.25165 /$ j.ijabe.20171006.3531

Citation: Liang K, Zhang L L, Lu W, Okinda C S, Shen M X. Optimization of compression formulation and load of food-grade tracers for grain traceability using central composite design. Int J Agric \& Biol Eng, 2017; 10(6): 221-230.

\section{Introduction}

Grain production and consumption in China account

Received date: 2017-05-30 Accepted date: 2017-08-26

Biographies: Liang Kun, $\mathrm{PhD}$, Lecturer, research interests: non-detection and traceability of agricultural products, Email: lkbb2006@126.com; Zhang Lingling, undergraduate, research interests: non-detection and traceability of agricultural products, Email: zhangllling@126.com; Lu Wei, PhD, Associate Professor, research interests: robotics and nondestructive testing, Email: njaurobot@njau.edu.cn; Cedric Sean Okinda, PhD candidate, research interests: computer application in agriculture, Email: cedsean@hotmail.com.

*Corresponding author: Shen Mingxia, $\mathrm{PhD}$, Professor, research interests: intelligent measurement and control, No. 40, Dianjiangtai Road, Pukou District, Nanjing 210031, Jiangsu, China. Tel: +86-25-58606502, Email: mingxia@njau.edu.cn. for $30 \%$ of total grain consumption worldwide, and grain food safety is a key safety issue for food production. Cereal grains, including wheat, beans, rice and coarse grains, and other grains are defined as grain foods by the Food and Agriculture Organization (FAO $)^{[1]}$. Numerous food safety issues have occurred recently ${ }^{[2]}$, promoting the demand for traceability and transparency in foodstuffs, and thus, for tools applied in effective tracing and quality control from harvest to storage, and finally to transport ${ }^{[3]}$. To enable better tracking and tracing of agricultural food products, many countries have introduced stricter regulations and smarter industrial development strategies ${ }^{[4-6]}$. As an effective means to ensure food safety, a tracing system can effectively realize traceability and quality control from farm to table ${ }^{[7]}$. In a tracing 
system, identity preservation (IP) is a key technology during information flow to achieve the rapid and effective identification of product information ${ }^{[8]}$. Ensuring IP in grain traceability would increase consumers' confidence and provide an advantage in the market ${ }^{[9]}$. By marking and labeling food grains in a way that ensures IP, when agricultural product quality and safety incidents occur, it is possible to trace the original agricultural products rapidly and promptly, and the timely recall of substandard agricultural products could reduce the losses suffered by producers. Therefore, a tracing system with effective IP is important for promoting food grain safety.

Local labeling and marketability from harvest to final destination are necessary to ensure grain traceability during transportation. However, associating unique and accurate labels with these types of bulk products in order to facilitate the direct identification of different lots is difficult $^{[10]}$. Grains such as wheat and corn have small individual sizes but are handled in massive quantities. Moreover, grain from various origins is typically mixed according to its different uses and grades at multiple points in the supply chain, making it impossible to determine the point of origin ${ }^{[11]}$. To address this problem, some iron pellets with radio-frequency identification (RFID) technology were developed and applied to achieve grain traceability in continuous grain flows ${ }^{[12]}$. However, the use of RFID-based identification technology in grain traceability requires that it can be safely removed from the final product by specific tracing devices. The high costs associated with this process have limited its widespread application. Subsequently, food-grade tracers, such as pill-sized particles, were proposed as a safe and low-cost identification technology for grain traceability, followed by a series of studies on food grain tracers. The earliest food-grade tracers were made of semolina using a pellet mill. This tracer was $6 \mathrm{~mm}$ in length and $4 \mathrm{~mm}$ in diameter, and it weighed $87 \mathrm{mg}^{[13]}$. Food-grade tracers, such as starch- or sugar-based pharmaceutical caplets with a printed barcode on the surface, have been developed. However, such starch-based tracers tend to absorb water, and their hardness declines as more water is absorbed, making them unsuitable for grain traceability applications during storage and transport ${ }^{[14]}$. Subsequently, a coating solution was applied to celluloseand sugar-based tracers to prevent damage and moisture absorption, and the effects of different types of tracers and coating materials on the tracers' physical properties were assessed ${ }^{[15]}$. Based on that study, food-grade tracers with a Data Matrix code printed on their surface were developed. The ruggedness of the code on the surface against abrasion and impact, as well as the effect of moisture conditioning, was assessed ${ }^{[16,17]}$

In a grain tracing system, it is necessary to assess the characterization of food-grade tracers in order to maintain the shape of the food-grade tracer and to avoid damage to the codes marked on the surface of a tracer. During the processes of harvesting, transportation, drying and storage, the food-grade tracers will be compressed by the load and can be deformed or even broken. Some researchers have investigated grains' mechanical characteristics to avoid damage during harvesting, handling, grading, and transporting ${ }^{[18-20]}$. Food-grade tracers also experience damage and friction during grain storage and transportation. Production process parameters will affect the mechanical characteristics of tracers and, thereby, the subsequent coating and printing process. However, little research has been devoted to assessing the effects of the production process parameters on the characterization of food-grade tracers. The food-grade grain tracers are generally made through a procedure involving compression, coating and printing. Direct compression is a simple and efficient method for compacting the premixed excipient directly to produce tracers ${ }^{[15]}$. Coating tracers with materials such as edible shellac and hydroxypropyl methylcellulose can improve their surface characteristics and minimize mechanical damage $e^{[15]}$. During printing, a readable code with traceability information is marked on the surface of tracers by an ink jet printer ${ }^{[16,17]}$. Among these three procedures, the compressing process has the most important effect on the mechanical characteristics of the tracers. In this process, the pressure of the tablet press machine and the formulation are the main factors that affect the mechanical characteristics of the tracers. Moreover, magnesium stearate is a common lubricant 
used in formulations, and its concentration also impacts the mechanical properties of the compressed tablets.

Therefore, in this work, we investigated the influences of tracer production process parameters (i.e., direct compressible formulation and compression load) on the impact characteristics (i.e., the breaking rate), compression characteristics (i.e., the peak shear force) and frictional characteristics (i.e., the friction coefficient) of food-grade tracers. The response surface methodology with a central composite design (CCD) approach was used to establish the relationship between the response of the tracer characteristics measured and the optimized pressure level and magnesium stearate content in the formulation. This research provides a reference for low-cost, environmentally adaptable and easily applied production technologies for the development of food-grade tracers in grain supply chains.

\section{Materials and methods}

\subsection{Materials}

The excipient selection for a direct compression formulation was performed based on the powder characteristics $^{[21]}$. Table 1 shows the four types of powder formulations, each of which consists of a mixture of three or four of the following powders: microcrystalline cellulose 102, pregelatinized starch, and magnesium stearate (Anhui Sunhere Pharmaceutical Excipients Co., Ltd., China), as well as lactose 80 mesh and lactose 100 mesh (Zhenjiang Kangfu Biological Engineering Co., Ltd., China). A sample of each type of formulation (approximately $100 \mathrm{~g}$ ) was mixed with a trough-type mixer (CH10, Tianfeng Pharmaceutical Machinery Co., Ltd., Shanghai, China) for $0.5 \mathrm{~h}$. The powder characteristics (i.e., the angle of repose, angle of spatula, compressibility, uniformity and flowability index) of the four types of mixed powder were determined by an intelligent powder physical property tester (BT-1001, Dandong Bettersize Instruments Ltd., Dandong, China). The two formulations with the highest flowability index also had the best powder flowability and therefore were selected for the following optimization experiments.

Table 1 Four grain tracer formulations

\begin{tabular}{lccc}
\hline \multicolumn{1}{c}{ Component } & Formulation \#1 & Formulation \#2 & Formulation \#3 \\
\hline Lactose 80 mesh/\% & 35.00 & 0.00 & 38.00 \\
Lactose 100 mesh/\% & 0.00 & 35.00 & 0.00 \\
Microcrystalline cellulose 102/\% & 64.50 & 64.50 & 5.00 \\
Pregelatinized starch/\% & 0.00 & 0.00 & 50.00 \\
Magnesium stearate/\% & 0.50 & 0.50 & 11.00 \\
\hline
\end{tabular}

\subsection{Tracer production}

Each selected formulation (without magnesium stearate) was mixed thoroughly using the trough-type mixer for $15 \mathrm{~min}$ and then mixed again for $3 \mathrm{~min}$ after adding magnesium stearate in different proportions. The total weight of each formulation powder was $1000 \mathrm{~g}$. A rotary tablet press (ZPS8, Shanghai Tianxiang Chentai Pharmaceutical Machinery Co., Ltd., Shanghai, China) was used to compress the two tracer formulations. The tracers were designed to be round particles so that they would be appropriate for printing QR codes on the surface in the future. The tracers were $11 \mathrm{~mm}$ in diameter and $5 \mathrm{~mm}$ in thick (Figure 1). The pressure of the tablet press machine is an important factor affecting the properties of grain tracers, which include the hardness of the tracers. In general, the greater the pressure is, the stronger and harder the tablet is ${ }^{[22]}$. Currently, in the tablet manufacturing process, the pressure for tablet production is dynamic, and measuring the pressure is difficult. In addition, the pressure will change when using different equipment, different tablet formulations and different tablet thicknesses. To adjust the pressure in the production process, Sacchetti et al. ${ }^{[23]}$ adjusted the pressure of the tablet machine to ensure the appropriate hardness value. A tablet hardness tester (YPD-300D, Shanghai Huanghai Medicine Checking Instrument Co., Ltd., Shanghai, China) was used to indirectly measure the average pressure. The pressure of the tablet press was constantly adjusted, and the hardness of the tablet was measured by the hardness tester until the hardness of the tablet reached the required pressure. To maintain a constant pressure, the hardness was checked every $5 \mathrm{~min}$ 
to ensure that the hardness of tablet did not fluctuate by more than $1 \mathrm{kgf}$.
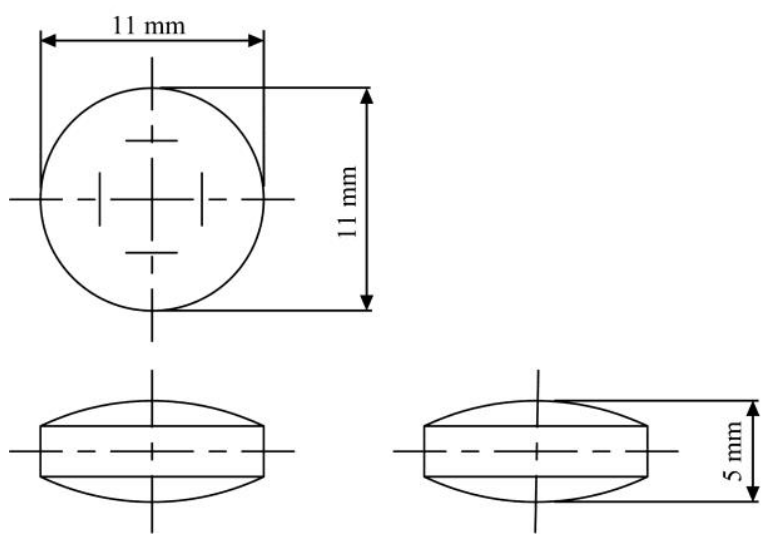

Figure 1 Dimensions of the food-grade tracer

\subsection{Tracer characterization test}

\subsubsection{Breaking rate determination}

The breaking rate of the grain tracers was estimated using 100 tracer samples by counting the number of damaged tracers after an impact test. Tracer samples were dropped from a 17-m high stairwell onto a concrete surface and then collected to determine the number of damaged tracers and calculate the breaking rate by Equation (1):

$$
Y_{1}=\frac{N_{1}}{N} \times 100 \%
$$

where, $N_{1}$ is the number of cracked samples after the impact test; $N$ is the total number of tracer samples, and $Y_{1}$ is the breaking rate.

\subsubsection{Peak shear force determination}

A shear test was conducted using a texture analyzer (TMS-PRO, Food Technology Corporation, USA) to measure the shear force of the food-grade tracer samples. A CA-1 single blade shear cell was fitted to a texture analyzer equipped with a $1000 \mathrm{~N}$ load cell. The trigger force was $0.5 \mathrm{~N}$. Each sample was subjected to a test speed of $30 \mathrm{~mm} / \mathrm{min}$ and a post-test speed of $80 \mathrm{~mm} / \mathrm{min}$. The peak shear force was calculated automatically by the software associated with the equipment based on the force-displacement plot. For each treatment in the experimental design, twenty independent measurements were performed according to American Society of Agricultural Engineers (ASAE) standard S368 ${ }^{[24]}$, and the average value was determined.

\subsubsection{Friction coefficient determination}

Predicting the friction coefficients of grains, forage materials, and other agricultural products on wood, metal and other structural surfaces is necessary for harvesting, storing and transporting, etc. ${ }^{[25]}$. As described by Yang et al. ${ }^{[26]}$, the experimental setup to measure the dynamic coefficients of friction between food-grade tracers and wheat was a coefficient of friction tester (MXD-01, Labthink Instruments Co., Ltd., Jinan, China). A single layer of wheat grain was evenly fixed on one side of a double-sided foam adhesive, and the other side was fixed on the test bench of the friction coefficient meter. Three tracers were placed into the grooves of the customized slider, ensuring that the wheat was in contact with the tracers. The slider was driven by a traction device to slide at a constant speed of $100 \mathrm{~mm} / \mathrm{min}$ over the surface of the wheat. Each experiment was repeated three times, and the average value was calculated.

\subsection{Experimental design and statistical analysis}

The pressure and magnesium stearate ratio in the formulation are the important factors during the production process that affect the performance of food-grade tracers. Pressure will affect the tablet appearance $^{[27]}$ : the greater the pressure, the smoother and more solid the tracers, thereby providing better mechanical properties, such as impact, compression, and frictional characteristics. As a commonly used lubricant, magnesium stearate can improve the flowability of powders $^{[28]}$ and smooth the tracers. However, increasing the concentration of magnesium stearate will also affect the tensile strength of the tablets ${ }^{[29]}$. Thus, the magnesium stearate ratio has a significant influence on the mechanical characteristics of tracers. Optimizing the pressure and magnesium stearate ratio can afford tracers with better mechanical properties.

The CCD of RSM was used to analyze and optimize the two factors (i.e., the magnesium stearate ratio $\left(X_{1}\right)$ and the pressure $\left(X_{2}\right)$ ) (Table 2). The impact, compression and frictional characteristic parameters of the breaking rate $\left(Y_{1}\right)$, peak shear force $\left(Y_{2}\right)$ and friction coefficient $\left(Y_{3}\right)$, respectively, were dependent variables. $\mathrm{A}$ generalized linear model with a binomial response distribution was employed, as shown in Equation (2):

$$
Y=b_{0}+b_{1} X_{1}+b_{2} X_{2}+b_{11} X_{1}^{2}+b_{22} X_{2}^{2}+b_{12} X_{1} X_{2}
$$


where, $Y$ is the response; $X_{1}$ and $X_{2}$ are the variables; $b_{0}$ is the constant; $b_{1}$ and $b_{2}$ are the coefficients of the linear terms; $b_{11}$ and $b_{22}$ are the coefficients of the quadratic terms, and $b_{12}$ is the coefficient of the interaction terms. According to the CCD, thirteen treatments were conducted (Table 5). Statistical analysis and regression models were performed with the help of the Design Expert package (Version 8.0.6, Stat-Ease, Inc., Minneapolis, MN, USA).

Table 2 Independent variables and their levels for CCD

\begin{tabular}{|c|c|c|c|c|c|c|}
\hline \multirow{2}{*}{ Variables } & \multirow{2}{*}{ Symbol } & \multicolumn{5}{|c|}{ Levels } \\
\hline & & $-\alpha$ & -1 & 0 & 1 & $\alpha$ \\
\hline $\begin{array}{c}\text { Magnesium stearate } \\
\text { ratio/\% }\end{array}$ & $X_{1}$ & 0.59 & 1.00 & 2.00 & 3.00 & 3.41 \\
\hline Pressure/kgf & $X_{2}$ & 3.93 & 6.00 & 11.00 & 16.00 & 18.07 \\
\hline
\end{tabular}

\section{Results and discussion}

\subsection{Powder physical property measurements}

Table 3 shows the powder physical properties measured for the four types of formulations. Powder flowability was evaluated using Carr's flowability index, which was calculated using the angle of repose, angle of spatula, compressibility and uniformity ${ }^{[30]}$. Four tests were conducted to measure the angle of repose, angle of spatula, compressibility and uniformity for the four formulations. Each examination was assigned 25 scores, and the flowability index was then calculated using the point scores out of $100^{[31]}$. A point in the range of '90-100' represents 'excellent' flowability, '80-89' represents 'good' flowability, '70-79' represents 'fair' flowability, '60-69' represents 'passable' flowability, ‘40-49' represents 'poor' flowability, '20-39' represents 'very poor' flowability, and '0-19' represents 'extremely poor' flowability ${ }^{[32]}$. All four formulations had passable powder flowability. Higher values of Carr's flowability index reflected better flowability ${ }^{[33]}$. Therefore, Formulations \#2 and \#4 had the best powder flowability, and they were used in the following experiments.

Table 3 Flowability indexes of the four types of food-grade tracer formulations

\begin{tabular}{|c|c|c|c|c|c|}
\hline Formulation & Angle of repose $/\left(^{\circ}\right)$ & Angle of spatula $/\left(^{\circ}\right)$ & Compressibility/\% & Uniformity & Flowability index \\
\hline Formulation \#1 & 36.28 & 56.18 & 28.99 & 5.42 & 67.00 \\
\hline Formulation \#2 & 37.22 & 49.71 & 27.27 & 5.52 & 68.00 \\
\hline Formulation \#3 & 36.07 & 48.96 & 31.17 & 5.88 & 65.50 \\
\hline Formulation \#4 & 37.56 & 48.93 & 27.78 & 5.96 & 68.00 \\
\hline
\end{tabular}

\subsection{Results of CCD experimental design}

As a frequently used lubricant, magnesium stearate is applied to improve the quality and production efficiency of tablets using the direct powder compression method ${ }^{[34]}$; however, its use can lead to issues such as reduced tracer hardness. The formulations were slightly changed by altering the magnesium stearate ratio in order to evaluate the effect of the magnesium stearate concentration on the impact characteristics (the breaking rate), compression characteristics (the peak shear force) and frictional characteristics (the friction coefficient) of food-grade tracers. The levels of magnesium stearate in Formulations \#2 and \#4 are shown in Table 4. The characteristics of the grain tracers of Formulations \#2 and \#4 subjected to 13 sets respectively are listed in Table 5. For Formulation \#2, the breaking rate varied from $34.00 \%$ to $100.00 \%$, the peak shear force ranged from $24.80 \mathrm{~N}$ to $139.10 \mathrm{~N}$, and the friction coefficient varied from 0.14 to 0.20 . For Formulation \#4, the breaking rate varied from $46.00 \%$ to $100.00 \%$, the peak shear force ranged from $18.00 \mathrm{~N}$ to $96.20 \mathrm{~N}$, and the friction coefficient varied from 0.14 to 0.32 .

\subsection{Establishment of regression models}

Based on the design and results of the CCD tests shown in Table 5, Design Expert software was used to fit the regression models of the breaking rate, peak shear force and friction coefficient for Formulations \#2 and \#4. The regression equations are listed in Table 6.

Analysis of variance (ANOVA) was used to analyze the models for significance and the suitability of the terms. The $p$-values, coefficients of determination $\left(R^{2}\right)$, adjusted $R^{2}$ values and adequate precisions of all the response models are shown in Table 7. For Formulation $\# 2$, the $p$-value of the friction coefficient $\left(Y_{3}\right)$ was 0.0148 , whereas those of the others $\left(Y_{1}, Y_{2}\right)$ were less than 0.01 . Thus, the model of the friction coefficient was significant, 
and the others were extremely significant at a $95 \%$ confidence level. Values of $R^{2}$ exceeding 0.8 indicated that the models of the breaking rate $\left(Y_{1}\right)$, peak shear force $\left(Y_{2}\right)$ and friction coefficient $\left(Y_{3}\right)$ fitted the experimental results well. The adequate precisions of the models were greater than 4 , indicating that the models were all adequate. For Formulation \#4, the $p$-values were all less than 0.01 , revealing that the models of the breaking rate $\left(Y_{1}\right)$, peak shear force $\left(Y_{2}\right)$ and friction coefficient $\left(Y_{3}\right)$ were extremely significant at a $95 \%$ confidence level. The $R^{2}$ values exceeded 0.8 , and the adequate precision values were greater than 4 . The regression models of the breaking rate $\left(Y_{1}\right)$, peak shear force $\left(Y_{2}\right)$ and friction coefficient $\left(Y_{3}\right)$ for Formulation \#4 were all adequate.

Table 4 Compositions and proportions of Formulations \#2 and \#4 at different magnesium stearate ratios

\begin{tabular}{|c|c|c|c|c|c|c|}
\hline & \multirow{2}{*}{ Component } & \multicolumn{5}{|c|}{ Magnesium stearate ratio $\left(X_{1}\right)$} \\
\hline & & $-\alpha$ & -1 & 0 & 1 & $\alpha$ \\
\hline \multirow{3}{*}{$\begin{array}{l}\text { Formulation } \\
\qquad \# 2\end{array}$} & Magnesium stearate/\% & 0.59 & 1.00 & 2.00 & 3.00 & 3.41 \\
\hline & Lactose $100 \mathrm{mesh} / \%$ & 35.00 & 34.00 & 33.00 & 34.00 & 32.00 \\
\hline & Microcrystalline cellulose $102 / \%$ & 64.41 & 65.00 & 65.00 & 63.00 & 64.59 \\
\hline \multirow{4}{*}{$\begin{array}{l}\text { Formulation } \\
\qquad \# 4\end{array}$} & Magnesium stearate/\% & 0.59 & 1.00 & 2.00 & 3.00 & 3.41 \\
\hline & Lactose $100 \mathrm{mesh} / \%$ & 38.00 & 38.00 & 37.50 & 36.00 & 36.00 \\
\hline & Microcrystalline cellulose $102 / \%$ & 50.41 & 50.00 & 49.50 & 50.00 & 49.50 \\
\hline & Pregelatinized starch $/ \%$ & 11.00 & 11.00 & 11.00 & 11.00 & 11.00 \\
\hline
\end{tabular}

Table 5 Experimental design and results for Formulations \#2 and \#4

\begin{tabular}{|c|c|c|c|c|c|c|c|c|}
\hline \multirow{2}{*}{ Runs } & \multirow{2}{*}{$\begin{array}{c}\text { Magnesium } \\
\text { stearate ratio } \\
X_{1} / \%\end{array}$} & \multirow{2}{*}{$\begin{array}{c}\text { Average } \\
\text { pressure } \\
X_{2} / \mathrm{kgf}\end{array}$} & \multicolumn{3}{|c|}{ Formulation \#2 } & \multicolumn{3}{|c|}{ Formulation \#4 } \\
\hline & & & $\begin{array}{c}\text { Breaking rate } \\
Y_{1} / \%\end{array}$ & $\begin{array}{c}\text { Peak shear force } \\
Y_{2} / \mathrm{N}\end{array}$ & $\begin{array}{c}\text { Friction coefficient } \\
\qquad Y_{3}\end{array}$ & $\begin{array}{c}\text { Breaking rate } \\
Y_{1} / \%\end{array}$ & $\begin{array}{c}\text { Peak shear force } \\
Y_{2} / \mathrm{N}\end{array}$ & $\begin{array}{c}\text { Friction coefficient } \\
\qquad Y_{3}\end{array}$ \\
\hline 1 & 1.00 & 6.00 & 93.00 & 48.80 & 0.16 & 99.00 & 40.60 & 0.25 \\
\hline 2 & 3.00 & 6.00 & 88.00 & 39.00 & 0.16 & 98.00 & 37.60 & 0.18 \\
\hline 4 & 3.00 & 16.00 & 47.00 & 91.30 & 0.14 & 66.00 & 57.40 & 0.16 \\
\hline 5 & 0.59 & 11.00 & 73.00 & 78.90 & 0.15 & 78.00 & 71.30 & 0.18 \\
\hline 6 & 3.41 & 11.00 & 72.00 & 68.00 & 0.14 & 70.00 & 51.20 & 0.14 \\
\hline 7 & 2.00 & 3.93 & 100.00 & 24.80 & 0.20 & 100.00 & 18.00 & 0.32 \\
\hline 8 & 2.00 & 18.07 & 34.00 & 139.10 & 0.14 & 46.00 & 85.80 & 0.16 \\
\hline 9 & 2.00 & 11.00 & 75.00 & 80.80 & 0.14 & 71.00 & 61.90 & 0.14 \\
\hline 10 & 2.00 & 11.00 & 69.00 & 73.80 & 0.14 & 80.00 & 61.40 & 0.15 \\
\hline 11 & 2.00 & 11.00 & 59.00 & 79.80 & 0.15 & 84.00 & 57.50 & 0.18 \\
\hline 12 & 2.00 & 11.00 & 68.00 & 83.50 & 0.14 & 67.00 & 62.50 & 0.16 \\
\hline 13 & 2.00 & 11.00 & 84.00 & 74.20 & 0.14 & 74.00 & 61.60 & 0.17 \\
\hline
\end{tabular}

Table 6 Response values corresponding to the regression equations

\begin{tabular}{ccc}
\hline & Response value $(Y)$ & Regression equation \\
\hline $\begin{array}{c}\text { Formulation } \\
\# 2\end{array}$ & $Y_{1} / \%$ & $Y_{1}=+112.50903-3.62678 X_{1}-2.25845 X_{2}-0.30000 X_{1} X_{2}+1.18750 X_{1}^{2}-0.062500 X_{2}^{2}$ \\
& $Y_{2} / \mathrm{N}$ & $Y_{2}=-8.12594+14.36813 X_{1}+7.24792 X_{2}-0.33000 X_{1} X_{2}-3.98500 X_{1}^{2}+0.010600 X_{2}^{2}$ \\
\hline$Y_{3}$ & $Y_{3}=+0.22561+3.23223 \times 10^{-3} X_{1}-0.012391 X_{2}-1.00000 ? 10^{-3} X_{1} X_{2}+8.75000 \times 10^{-4} X_{1}^{2}+5.35000 ? 10^{-4} X_{2}^{2}$ \\
\hline $\begin{array}{c}\text { Formulation } \\
\# 4\end{array}$ & $Y_{1} / \%$ & $Y_{1}=+128.72300-8.08921 X_{1}-4.45119 X_{2}-0.050000 X_{1} X_{2}+1.71250 X_{1}^{2}+0.048500 X_{2}^{2}$ \\
& $Y_{2} / \mathrm{N}$ & $Y_{2}=-26.34144+8.99679 X_{1}+11.55479 X_{2}-1.79000 X_{1} X_{2}+0.47875 X_{1}^{2}-0.16785 X_{2}^{2}$ \\
& $Y_{3}$ & $Y_{3}=+0.52709-0.041821 X_{1}-0.047857 X_{2}+3.50000 ? 10^{-3} X_{1} X_{2}-3.12500 ? 10^{-3} X_{1}^{2}+1.47500 \times 10^{-3} X_{2}^{2}$ \\
\hline
\end{tabular}


Table 7 ANOVA results of Formulations \#2 and \#4 regarding the adequacy of the regression models

\begin{tabular}{|c|c|c|c|c|c|}
\hline & Responses & $p$-value & $R^{2}$ & $\operatorname{Adj}-R^{2}$ & Adeq Precision \\
\hline \multirow{3}{*}{ Formulation \#2 } & $Y_{1}$ & 0.0023 & 0.8988 & 0.8265 & 11.483 \\
\hline & $Y_{2}$ & 0.0002 & 0.9529 & 0.9192 & 17.198 \\
\hline & $Y_{3}$ & 0.0148 & 0.8217 & 0.6944 & 8.337 \\
\hline \multirow{3}{*}{ Formulation \#4 } & $Y_{1}$ & 0.0060 & 0.8645 & 0.7677 & 9.731 \\
\hline & $Y_{2}$ & $<0.0001$ & 0.9804 & 0.9665 & 29.696 \\
\hline & $Y_{3}$ & 0.0022 & 0.9001 & 0.8288 & 11.458 \\
\hline
\end{tabular}

\subsection{Response surface analysis of grain tracer properties}

\subsubsection{Property analysis of Formulation \#2}

The mutual interaction effects of the magnesium stearate ratio and pressure on the grain tracer properties of Formulation \#2 can be seen on the three-dimensional (3D) response surface plots in Figure 2. Figure 2a depicts the effects of the interaction between the magnesium stearate ratio and pressure on the breaking rate. The pressure significantly affected the breaking rate. Additionally, increasing the pressure resulted in a linear increase in the breaking rate, which might be explained by the powder being compacted more firmly as the pressure increased. The binding force of the powder was enhanced, the ability to resist external force increased, and thus, the breaking rate decreased. The magnesium stearate ratio exerted a slight effect on the breaking rate. The breaking rate of food-grade tracers gradually decreased as the magnesium stearate concentration increased.

Figure $2 b$ reveals the effects of the magnesium stearate ratio and pressure on the peak shear force of food-grade tracers. The peak shear force was observed to increase as the pressure increased. According to Lee et al. ${ }^{[14]}$, high pressure can lead to tightly compact tracer structures and increased shearing forces. The peak shear force was negatively correlated with the magnesium stearate concentration, and it decreased slightly as the magnesium stearate concentration increased.

The effects of the magnesium stearate ratio and pressure on friction coefficient are presented in Figure 2c. The friction coefficient decreased as the pressure increased until approximately $13.00 \mathrm{kgf}$. This behavior may be attributable to the fact that higher pressure increases the smoothness of the tracer ${ }^{[27]}$, leading to a decrease in the friction coefficient. The friction coefficient tended to increase when pressure exceeded $13.00 \mathrm{kgf}$. In contrast, the magnesium stearate ratio was negatively related to the friction coefficient at pressures of $11.00-16.00 \mathrm{kgf}$. One explanation is that magnesium stearate has good lubricity and acts to smooth the surface of the tracer, thereby reducing the friction coefficient.

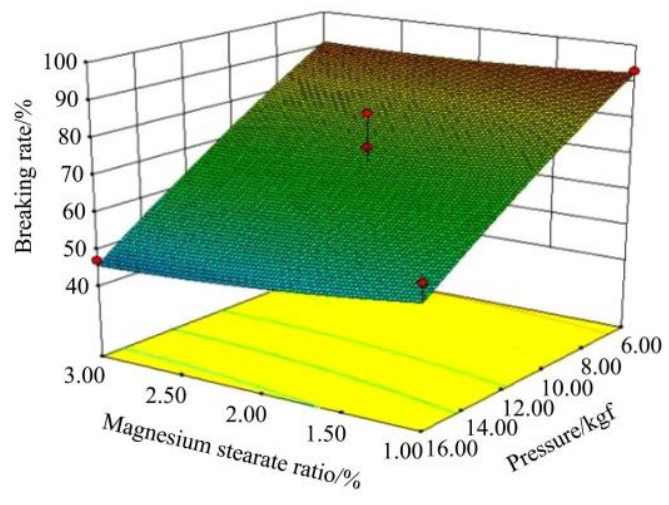

a. Breaking rate

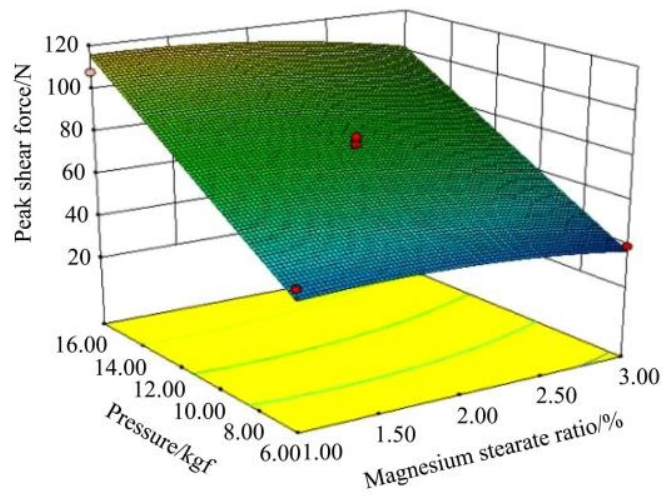

b. Peak shear force

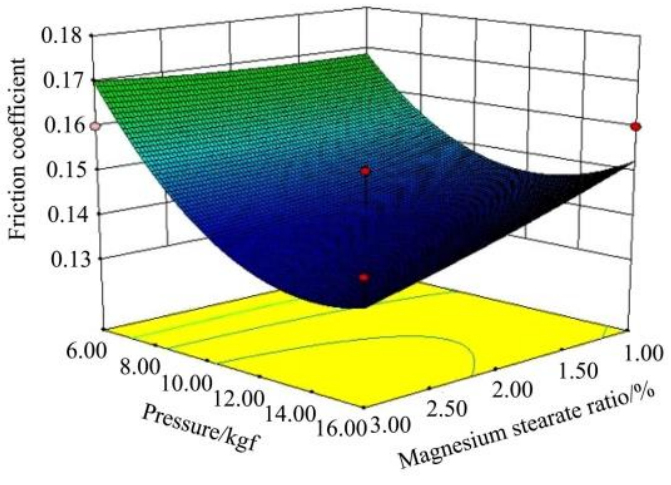

c. Friction coefficient

Figure 2 Effects of magnesium stearate ratio and pressure on physical properties of grain tracers of Formulation \#2 


\subsubsection{Property analysis of Formulation \#4}

The mutual interaction effects of the magnesium stearate ratio and pressure on the grain tracer properties of Formulation \#4 can be seen on the 3D response surface plots in Figure 3. The effects of the magnesium stearate concentration and pressure on the breaking rate are presented in Figure 3a. The pressure significantly influenced the breaking rate of Formulation \#4, as the same manner observed from Formulation \#2. The breaking rate decreased linearly as the pressure increased and decreased slowly as the magnesium stearate concentration increased.

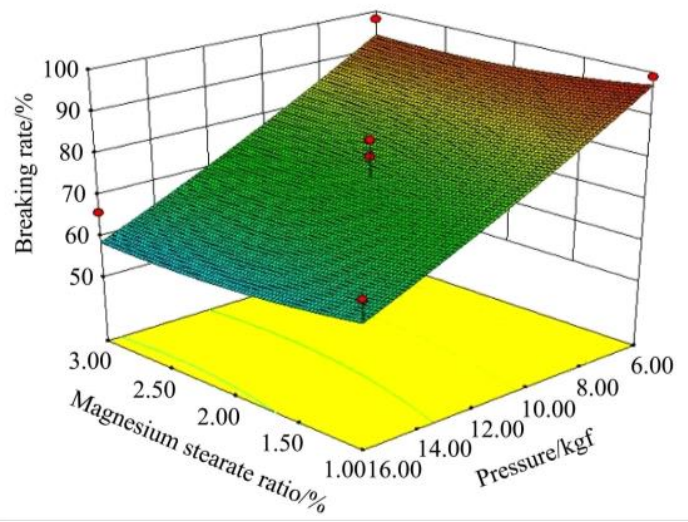

a. Breaking rate

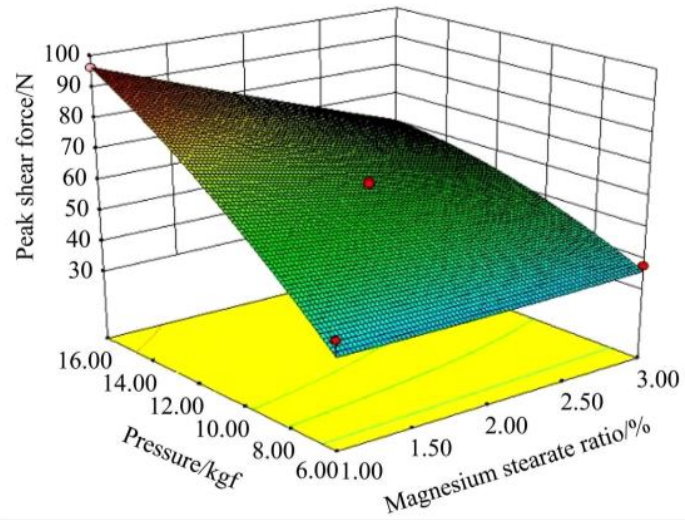

b. Peak shear force

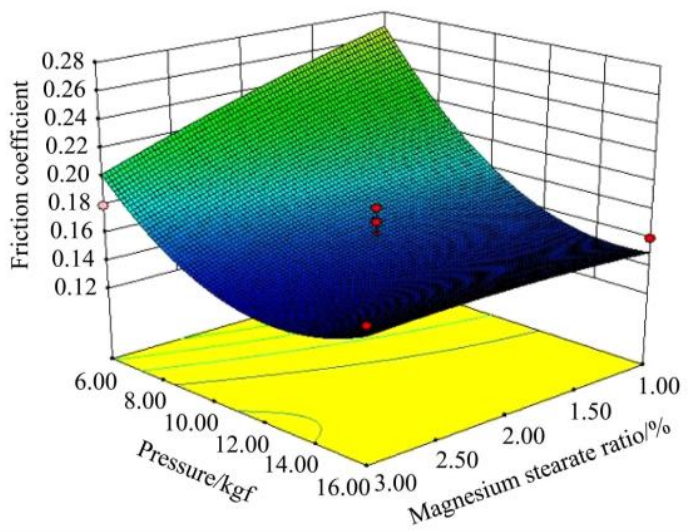

c. Friction coefficient

Figure 3 Effects of the magnesium stearate ratio and pressure on the physical properties of grain tracers of Formulation \#4
Figure $3 b$ reveals the effects of the magnesium stearate ratio and pressure on the peak shear force. The peak shear force increased sharply as the pressure increased while the magnesium stearate ratio ranged from $1.00 \%$ to $2.00 \%$. Additionally, the magnesium stearate ratio did not affect the peak shear force significantly at pressures of 6.00-10.00 kgf. However, when the pressure exceeded $10.00 \mathrm{kgf}$, the peak shear force decreased as the magnesium stearate ratio increased.

Figure $3 \mathrm{c}$ shows the effects of the magnesium stearate ratio and pressure on the friction coefficient. The friction coefficient decreased as the pressure increased, but this tendency diminished when the pressure reached approximately $12.00 \mathrm{kgf}$ and then tended to increase. When the pressure ranged from $6.00 \mathrm{kgf}$ to $11.00 \mathrm{kgf}$, the friction coefficient decreased as the magnesium stearate ratio increased. However, when the pressure exceeded $11.00 \mathrm{kgf}$, the friction coefficient exhibited no obvious change as the magnesium stearate ratio increased.

\subsection{Optimization response and verification test}

The aim of this investigation was to determine the optimal process parameters required to achieve better tracer characteristics. The optimization process was conducted using Design Expert software, assigning constraints 'in the range' for independent variables, 'minimized' for the breaking rate and friction coefficient responses, and 'maximized' for the peak shear force. The optimal conditions recommended by the software were a magnesium stearate ratio of $2.25 \%$ and a pressure of $16.00 \mathrm{kgf}$ for Formulation \#2. The optimal Formulation \#2 was adjusted to $33.00 \%$ lactose 100 mesh, $64.75 \%$ microcrystalline cellulose 102 and $2.25 \%$ magnesium stearate. Under the optimal conditions, the breaking rate $47.42 \%$, peak shear force $110.82 \mathrm{~N}$, and friction coefficient 0.140 were obtained. For Formulation \#4, the optimal conditions were found to be a magnesium stearate ratio of $1.02 \%$ and a pressure of $16.00 \mathrm{kgf}$, in which case the breaking rate was $62.63 \%$, the peak shear force was $96.00 \mathrm{~N}$, and the friction coefficient was 0.150 . The optimal Formulation \#4 was adjusted to $38.00 \%$ lactose 100 mesh, 49.98\% microcrystalline cellulose 102, $11.00 \%$ pregelatinized starch and $1.02 \%$ magnesium 
stearate. Confirmation experiments were performed under the optimal conditions to verify the accuracy of the models. The experimental and predicted values are tabulated in Table 8. Comparing the experimental and predicted results revealed that they were relatively similar, indicating that the models were highly reliable. This optimization study revealed the pressure and formulation suitable for food-grade tracer production and can serve as a reference for the study and application of the tracing system.

Table 8 Comparison of the predicted and experimental values of the responses

\begin{tabular}{|c|c|c|c|c|c|c|}
\hline \multirow{2}{*}{ Responses } & \multicolumn{3}{|c|}{ Formulation \# 2} & \multicolumn{3}{|c|}{ Formulation \# 4} \\
\hline & Predicted & Experimental & Error & Predicted & Experimental & Error \\
\hline Breaking rate $/ \%$ & 47.42 & 43.00 & 0.1027 & 62.63 & 55.00 & 0.1387 \\
\hline Peak shear force/N & 110.82 & 104.00 & 0.0656 & 96.00 & 88.20 & 0.0884 \\
\hline Friction coefficient & 0.140 & 0.133 & 0.0526 & 0.150 & 0.157 & 0.0446 \\
\hline
\end{tabular}

Analyzing the experimental results showed that the values of the breaking rate and friction coefficient were smaller and that the values of the peak shear force of Formulation \#2 were larger than those of Formulation \#4. Therefore, Formulation \#2 is more suitable for grain tracer production.

\section{Conclusions}

This study evaluated the effects of production parameters on the impact, compression, and frictional characteristics of food-grade tracers and optimized the process using the CCD of RSM. Among the four formulations, Formulations \#2 (consisting of $35.00 \%$ lactose 100 mesh, $64.50 \%$ microcrystalline cellulose 102 and $0.50 \%$ magnesium stearate) and \#4 (consisting of $38.00 \%$ lactose 100 mesh, $50.00 \%$ microcrystalline cellulose 102, $11.00 \%$ pregelatinized starch and $1.00 \%$ magnesium stearate) were selected for subsequent optimization experiments because of their superior flowability compared to the other formulations tested. Empirical mathematical models relating the magnesium stearate ratio and pressure to the responses of the breaking rate, peak shear force and friction coefficient were established. The optimal production conditions of food-grade tracers were found to be a magnesium stearate ratio of $2.25 \%$ and pressure of $16.00 \mathrm{kgf}$ for Formulation \#2 and a magnesium stearate ratio of $1.02 \%$ and pressure of $16.00 \mathrm{kgf}$ for Formulation \#4. Under the optimal conditions, Formulation \#2 consisted of $33.00 \%$ lactose 100 mesh, $64.75 \%$ microcrystalline cellulose 102 and 2.25\% magnesium stearate, and Formulation \#4 consisted of $38.00 \%$ lactose 100 mesh, $49.98 \%$ microcrystalline cellulose $102,11.00 \%$ pregelatinized starch and $1.02 \%$ magnesium stearate. The experimental response values were extremely close to the predicted values obtained from the fitted mathematic models. The experimental results also showed that Formulation \#2 was more suitable for grain tracer production than Formulation \#4 because of its superior characteristics.

\section{Acknowledgments}

This work was supported by the National Natural Science Foundation of China (31401610), the Fundamental Research Funds for the Central Universities of China (KJON201557), the Outstanding Youth Foundation Science and Technology Fund of College of Engineering at Nanjing Agricultural University (YQ201603), and the Jiangsu Agriculture Science and Technology Innovation Fund (CX(16)1059).

\section{[References]}

[1] Zhu H, Jackson P, Wang W. Consumer anxieties about food grain safety in China. Food Control, 2017; 73: $1256-1264$.

[2] Aung M M, k Chang Y S. Traceability in a food supply chain: Safety and quality perspectives. Food Control, 2014; 39: 172-184.

[3] Wang X, He Q, Matetic M, Jemric T, Zhang X. Development and evaluation on a wireless multi-gas-sensors system for improving traceability and transparency of table grape cold chain. Computers and Electronics in Agriculture, 2017; 135: 195-207.

[4] Narsimhalu U, Potdar V, Kaur A. A case study to explore influence of traceability factors on Australian food supply chain performance. Procedia - Social and Behavioral Sciences, 2015; 189: 17-32. 
[5] Can-Trace. Can-Trace develops traceability standards for all food products sold in Canada. www.can-trace.org. 2003. Accessed on [2017-05-21].

[6] EU. (EC) No. 178/2002 of the european parliament and of the council of 28 January 2002. Official Journal of the European Communities, 2002; L31: 1-24.

[7] Ceruti F C, Lazzari S, Lazzari F A. Traceability in the wheat production chain. 9th International Working Conference on Stored Product Protection, 2006; pp.1198-1205.

[8] Lee K M, Armstrong P R, Thomasson J A, Sui R X, Casada M, Herrman T J. Application of binomial and multinomial probability statistics to the sampling design process of a global grain tracing and recall system. Food Control, 2011; 22(7): 1085-1094.

[9] Herrman T J. White paper on traceability in the U. S. grain and plant protein feed ingredient industries.

[10] Comba L, Belforte G, Dabbene F, Gay P. Methods for traceability in food production processes involving bulk products. Biosystems Engineering, 2013; 116(1): 51-63.

[11] Thakur M, Hurburgh C R. Framework for implementing traceability system in the bulk grain supply chain. Journal of Food Engineering, 2009; 95(4): 617-626.

[12] Kvarnström B, Bergquist B, Vännman K. RFID to improve traceability in continuous granular flows-An experimental case study. Quality Engineering, 2011; 23(4): 343-357.

[13] Hirai Y, Schrock M D, Oard D L, Herrman T J. Delivery system of tracing caplets for wheat grain traceability. Applied Engineering in Agriculture, 2006; 22(5): 747-750.

[14] Sui R X, Thomasson J A, Herrman T. Development of tracers for grain tracing system. Asabe Annual International Meeting, 2007.

[15] Lee K M, Armstrong P R, Thomasson J A, Sui R X, Casada M, Herrman T J. Development and characterization of food-grade tracers for the global grain tracing and recall system. Journal of Agricultural and Food Chemistry, 2010; 58(20): 10945-10957.

[16] Liang K, Thomasson J A, Lee K M, Shen M X, Ge Y, Herrman T J. Printing data matrix code on food-grade tracers for grain traceability. Biosystems Engineering, 2012; 113(4): 395-401.

[17] Liang K, Thomasson J A, Shen M X, Armstrong P R, Ge Y, Lee $\mathrm{K} \mathrm{M}$, et al. Ruggedness of $2 \mathrm{D}$ code printed on grain tracers for implementing a prospective grain traceability system to the bulk grain delivery system. Food Control, 2013; 33(2): 359-365.

[18] Kamst G F, Bonazzi C, Vasseur J, Bimbenet J J. Effect of deformation rate and moisture content on the mechanical properties of rice grains. Transactions of the ASAE, 2002; 45(1): 145-151.

[19] Güzel E, Akçalı İ D, İnce A. Behavior of peanut bulk under static loads. Journal of Food Engineering, 2007; 80(2): 385-390.
[20] Subramanian S, Viswanathan R. Bulk density and friction coefficients of selected minor millet grains and flours. Journal of Food Engineering, 2007; 81(1): 118-126.

[21] Sanghvi P P, Collins C C, Shukla A J. Evaluation of Preflo modified starches as new direct compression excipients. I. Tabletting characteristics. Pharmaceutical Research, 1993; 10(11): 1597-1603.

[22] Zhang Y L, Law Y, Chakrabarti S. Physical properties and compact analysis of commonly used direct compression binders. AAPS PharmSciTech, 2003; 4(4): 489-499.

[23] Sacchetti M, Teerakapibal R, Kim K, Elder Jr E J. Role of water sorption in tablet crushing strength, disintegration, and dissolution. AAPS Pharmscitech, 2017; 1-13.

[24] ASAE Standard. Compression test of food materials of Convex Shape. ASAE S368.4 DEC2000, 2008.

[25] Shafaei S M, Nourmohamadi-Moghadami A, Kamgar S. Analytical study of friction coefficients of pomegranate seed as essential parameters in design of post-harvest equipment. Information Processing in Agriculture, 2016; 3(3): 133-145.

[26] Yang Z M, Guo Y M, Cui Q L, Li H B. Test and influence factors analysis of friction characteristics of millet. Transactions of the CSAE, 2016; 32(16): 258-264. (in Chinese)

[27] Matsumoto R, Kawakami K, Aoki S. Impact of compression pressure on tablet appearance. International Journal of Pharmaceutics, 2007; 341(1-2): 44-49.

[28] Faqih A M N, Mehrotra A, Hammond S V, Muzzio F J. Effect of moisture and magnesium stearate concentration on flow properties of cohesive granular materials. International Journal of Pharmaceutics, 2007; 336(2): 338-345.

[29] Iranloye T, Parrott E. Effects of compression force, particle size, and lubricants on dissolution rate. J Pharm Sci., 1978; 67(4): 535-539.

[30] Ganesan V, Rosentrater K A, Muthukumarappan K. Physical and flow properties of regular and reduced fat distillers dried grains with solubles (DDGS). Food and Bioprocess Technology, 2009; 2(2): 156-166.

[31] Carr R. Evaluating flow properties of solids. Chem. Eng., 1965; 72(2): 163-168.

[32] Otsuka T, Iwao Y, Miyagishima A, Itai S. Application of principal component analysis enables to effectively find important physical variables for optimization of fluid bed granulat or conditions. International Journal of Pharmaceutics, 2011; 409(1-2): 81-88.

[33] Sakurai Y, Mise R, Kimura S, Noguchi S, Iwao Y, Itai S. Novel method for improving the water dispersibility and flowability of fine green tea powder using a fluidized bed granulator. Journal of Food Engineering, 2017; 206: 118-124.

[34] Nakamura S, Yamaguchi S, Hiraide R, Iga K, Sakamoto T, Yuasa H. Setting ideal lubricant mixing time for manufacturing tablets by evaluating powder flowability. AAPS Pharmscitech, 2017; pp.1-9. 